\title{
Nonlinear Regression Analysis to Determine Infection Models of Colletotrichum acutatum Causing Anthracnose of Chili Pepper Using Logistic Equation
}

\author{
Wee Soo Kang1, Sung Chul Yun² and Eun Woo Park ${ }^{1 *}$ \\ ${ }^{1}$ Department of Agricultural Biotechnology, Seoul National University, Seoul 151-921, Korea \\ ${ }^{2}$ Department of Biomedical Sciences, Sun Moon University, Asan 330-744, Korea \\ (Received on November 16, 2009; Accepted on December 5, 2009)
}

\begin{abstract}
A logistic model for describing combined effects of both temperature and wetness period on appressorium formation was developed using laboratory data on percent appressorium formation of Colletotrichum acutatum. In addition, the possible use of the logistic model for forecasting infection risks was also evaluated as compared with a first-order linear model. A simplified equilibrium model for enzymatic reactions was applied to obtain a temperature function for asymptote parameter $(A)$ of logistic model. For the position $(B)$ and the rate $(k)$ parameters, a reciprocal model was used to calculate the respective temperature functions. The nonlinear logistic model described successfully the response of appressorium formation to the combined effects of temperature and wetness period. Especially the temperature function for asymptote parameter $A$ reflected the response of upper limit of appressorium formation to temperature, which showed the typical temperature response of enzymatic reactions in the cells. By having both temperature and wetness period as independent variables, the nonlinear logistic model can be used to determine the length of wetness periods required for certain levels of appressorium formation under different temperature conditions. The infection model derived from the nonlinear logistic model can be used to calculate infection risks using hourly temperature and wetness period data monitored by automated weather stations in the fields. Compared with the nonlinear infection model, the linear infection model always predicted a shorter wetness period for appressorium formation, and resulted in significantly under- and over-estimation of response at low and high temperatures, respectively.
\end{abstract}

Keywords : anthracnose, disease forecast, infection risk, logistic model, pepper, temperature function

Colletotrichum species causes significant economic losses on fruit crops by outbreaking anthracnose in the fruiting stage (Freeman et al., 1998; Kim, 1993; Park and Kim,

\footnotetext{
*Corresponding author.

Phone) +82-2-880-4672, FAX) +82-2-872-2317

E-mail)ewpark@snu.ac.kr
}

1992). In Korea, anthracnose caused about $13 \%$ losses of total pepper production during 1995 to 1999 (Shin et al., 1999). Of several species of Colletotrichum, C. capsici and C. gloeosporioides were known to be the main causal pathogens of pepper (Capsicum annuum) in Asia (Kim, 1993; Manandhar et al., 1995; Park and Kim, 1992; Shin et al., 1999). However, Kang et al. (2005) recently proved that C. acutatum was the major pathogen of pepper anthracnose in Korea by DNA homology comparison of ITS region and PCR analysis using species-specific primers.

Conidia of the anthracnose pathogen produced on diseased plant tissues are disseminated to pepper plants mainly by wind-blown rain. The conidia deposited on plant surface germinate and form appressoria prior to infection under favorable conditions. The pathogen requires wet conditions on the plant surface to infect host plants, and the length of wetness required for plant infection depends on temperature (Cho et al., 1987; Madden et al., 1996; Peres et al., 2005). Fungicides are commonly used to control the disease in the field, and regular sprays at 10-day intervals starting from early July until 7 days prior to harvest are recommended in Korea (Anonymous, 2008). However, unnecessary fungicides are often applied when weather conditions are not favorable.

Disease forecasts based on infection models support crop growers in timing the fungicide sprays only when the weather conditions are conducive for plant infections by fungal pathogens (Campbell and Madden, 1990). In many cases, infection models are polynomial equations describing the response to combined effects of temperature and wetness period (Biggs and Northover, 1988; Evans et al., 1992; Lalancette et al., 1988). In order to enhance the fitness of model, the response data and/or independent variables may be transformed prior to fitting the polynomial equations (Bulger et al., 1987; Hong and Hwang, 1998; Kim and Park, 1988; Schuh, 1991). However, the polynomial equations often do not reflect significance of biological processes. For example, the upper limit of response to temperature and wetness period could not be properly described by the polynomial equations (Scherm and van Bruggen, 1993). 
Intrinsically nonlinear growth models, such as the logistic and the Weibull functions, are also used to describe the disease response to temperature or wetness period but not both (Carisse et al., 1993; Duthie, 1997; Magboul et al., 1992). It is not common in nonlinear models to include both temperature and wetness period to explain their combined effects on disease development. A method for nonlinear description of a response to the combined effects of temperature and wetness period has been discussed by Hau et al. (1985): The time component (wetness period) is first analyzed separately for each temperature data subset and then calculated parameter values are used in a second analysis where temperature is the independent variable. Using this two-stage approach, Duthie (1997) proposed a nonlinear model for directly evaluating the response of foliar parasites to the combined effects of wetness period and temperature. In this study, we developed a logistic model to describe the combined effects of temperature and wetness period on the percent appressorium formation of $C$. acutatum using the two-stage approach by Hau et al. (1985). From the logistic model, a nonlinear infection model was derived to determine hourly infection risk levels.

\section{Materials and Methods}

Inoculum and pepper fruit preparation. An isolate of $C$. acutatum, which was obtained from the Fungal Disease Laboratory, Chungbuk National University (Kim, 1993), was cultured on potato dextrose agar for 3 to 7 days at $27^{\circ} \mathrm{C}$ under $12 \mathrm{hr}$ light-dark cycles. Conidial suspension was prepared by placing $3 \mathrm{~mL}$ of sterile distilled water on colony matrix and gently scraping colony surface with the bottom tip of 1.5-mL polypropylene microcentrifuge tube. The suspension was filtered through two layers of cheesecloth and adjusted to the concentration of $10^{6}$ conidia $/ \mathrm{mL}$ by adding sterile distilled water using a hemacytometer. Pepper plants (Capsicum annuum L., variety 'Dabotop') were grown in a greenhouse for about 18 weeks from sowing until the second branch stage at 20 to $25^{\circ} \mathrm{C}$. Immature green fruits were harvested when the length of fruits reached to their full length (approximately $10 \mathrm{~cm}$ ). The fruits were washed in running tap water and wiped dry with paper towels.

Inoculation and microscopic observation of appressorium formation. The detached pepper fruits were inoculated with the conidial suspension by spraying with an airbrush until runoff. Each of the inoculated fruits was suspended inside of a test tube ( $4 \mathrm{~cm}$ diameter) by the fruit stalk tied to a cross-shaped metal brace on the opening of test tube. In order to keep humid conditions inside the test tube, a piece of paper towel soaked in sterile distilled water was placed at the bottom of test tube without touching the pepper fruit and the tube was capped with aluminum foil. The test tubes with an inoculated pepper fruit were kept in incubators which were set at $20,23,25,28,30$, and $33^{\circ} \mathrm{C}$. After 0,6 , $12,15,18,21,24,30$, and 36 hours of incubation, the pepper fruit was taken out of the test tube and air-dried, and then the fruit surface was peeled off by a razor blade. Appressorium formation from germinating conidia on the fruit surface was observed under light microscope at 400X magnification without staining. One hundred conidia from 5 6 microscopic fields of views were examined for appressorium formation and the percentage of conidia forming appressoria was determined. If the original conidia from which appressoria were formed could not be distinguished because of too much hyphal growth, those conidia were excluded in the microscopic observation. For each combination of temperatures and incubation periods, three fruits were used as replications.

Model development. Effects of temperature and wetness period on appressorium formation were analyzed in two ways using linear and nonlinear regression. For linear regression, the STEPWISE procedure of SAS (SAS Institute Inc., 1985) was applied to select the most appropriate model using the first and second order terms of temperature $(T)$ and wetness period $(W)$ as independent variables. The best fit model was selected based on the lack-of-fit sum of squares and the coefficient of determination $\left(R^{2}\right)$. In the case of nonlinear regression analysis on the percent appressorium formation at each temperature, the NLIN procedure with the GAUSS iterative method (SAS Institute Inc., 1985) was applied to fit the logistic model in the following form:

$$
P=P(W)=\frac{A}{1+e^{B-k W}}
$$

where $P$ and $W$ represent the percent appressorium formation and wetness period, respectively. $A$ is the asymptote parameter and was fixed to the maximum percent of appressorium formation which was observed at each temperature. $B$ and $k$ are the parameters for position and rate of the model, respectively. $A, B$, and $k$ are functions of temperature.

The temperature functions for $A, B$, and $k$ were determined empirically using the parameter estimates in the logistic models for appressorium formation data at each temperature. The asymptotic levels of $A$ are affected by temperature and may reflect enzymatic activities in the fungal cells. Thus, an enzymatic activity model, the Equilibrium Model (Peterson et al., 2004), was applied to describe the effect of temperature on the maximum percent of appressorium formation of $C$. acutatum. The Equilibrium Model was 
originally developed to describe the velocity of catalytic activity of an enzyme affected by temperature over time (Peterson et al., 2004). By setting the time to zero in the model, the Equilibrium Model can be simplified as the following form:

$$
V_{\max }=\frac{k_{B} T e^{\left(-\frac{\left.\Delta G_{\text {ct }}^{\sharp}\right)}{R T} E_{0}\right.}}{h\left(1+e^{\left(\frac{\Delta H_{e q}\left(\frac{1}{T_{e q}}-\frac{1}{T}\right)}{R}\right)}\right)}
$$

where $k_{B}$ is Boltzmann's constant, $T$ is absolute temperature $(\mathrm{K}), h$ is Planck's constant, $R$ is ideal gas constant, and
$\Delta G_{\text {cat }}^{\ddagger}, E_{0}$, and $\Delta H_{e q}$ are the enthalpy energy constants related to the enzyme's reaction process. $T_{e q}$ is the absolute temperature $(\mathrm{K})$ where the amounts of active and inactive forms of an enzyme become equal.

In order to apply the simplified Equilibrium Model (Eq. 2) for describing the quantitative relationship between the asymptotic values $(A)$ of appressorium formation and temperature, the model was further simplified as the following form:

$$
A=A(T)=\frac{b_{0}(T+273.15) e^{\left(\frac{b_{1}}{T+273.15}\right)}}{1+e^{b_{2}\left(\frac{1}{T_{e q}}-\frac{1}{T+273.15}\right)}}
$$

where $b_{0}, b_{1}$, and $b_{2}$ are parameters to be estimated, and $T$ is
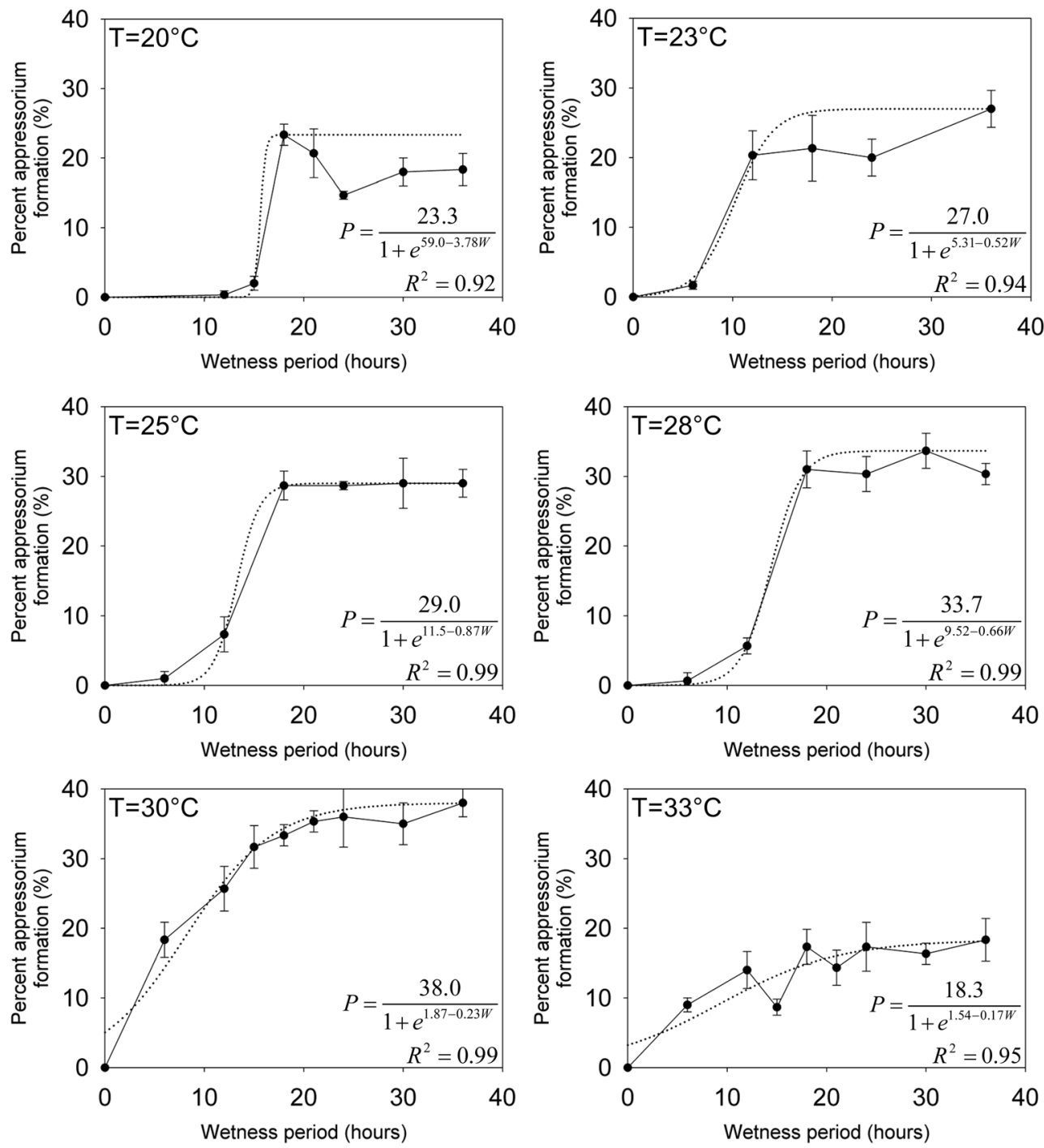

Fig. 1. Effects of wetness period on the percent appressorium formation of $C$. acutatum at different temperatures. The logistic model, shown in dotted line, was fitted to the observed data subset at each temperature. The asymptote parameter $(A)$ was fixed at the maximum percent appressorium formation. 
temperature $\left({ }^{\circ} \mathrm{C}\right)$. Assigning values of $T_{e q}$ from $293.15 \mathrm{~K}$ $\left(20.0^{\circ} \mathrm{C}\right)$ to $313.15 \mathrm{~K}\left(40.0^{\circ} \mathrm{C}\right)$ by an increment of $1.0 \mathrm{~K}$, nonlinear fittings were conducted. The model having the highest determination coefficient $\left(R^{2}\right)$ was selected to be the best model for the asymptote parameter.

The temperature functions for $B$ and $k$ were determined using nonlinear regression on the estimates of $B$ and $k$ of the logistic models for appressorium formation at each temperature. Considering the shapes of scattered diagrams of the estimates of $B$ and $k$ of the logistic models, the following forms of reciprocal equation were used in the model statement of the NLIN procedure with the GAUSS iteration method (SAS Institute Inc., 1985):

$$
\begin{aligned}
& B=B(T)=\frac{b_{3}}{T-b_{4}} \\
& k=k(T)=\frac{b_{5}}{T-b_{6}}
\end{aligned}
$$

where $b_{3}, b_{4}, b_{5}$, and $b_{6}$ are the parameters to be estimated.

Model evaluation. The linear and nonlinear models for the percent appressorium formation were evaluated with respect to two aspects: (1) the root mean square error (RMSE) of the differences between the observed and estimated percent appressorium formation, and (2) the possible levels of infection risks forecast based on hourly weather data. Using the percent appressorium formation data in Fig. 1, RMSEs for the linear and nonlinear models for each temperature were calculated as $R M S E=\sqrt{\sum\left(O_{i}-E_{i}\right)^{2} / n}$, where to $O_{i}$ and $E_{i}$ are the $i$ th data of observed and estimated percent appressorium formation, respectively, and $n$ is the number of observations.

The possible levels of infection risks were forecast by the linear and nonlinear models based on hourly weather data collected from an automated weather station (AWS) installed at the experimental pepper field of Sun Moon University in Asan, Korea in the growing seasons of 2005 and 2006. The AWS consisted of the CR10X data logger (Campbell Scientific, Inc., Logan, Utah, USA) and various sensors for measuring weather factors including temperature $\left({ }^{\circ} \mathrm{C}\right.$; HMP45C, Campbell Scientific, Inc., USA), wetness ( $\mathrm{hr} / \mathrm{hr}$; 237, Campbell Scientific, Inc., USA), rainfall (mm; TE525MM, Texas Electronics, Inc., Dallas, Texas, USA). The possible hourly infection risk $(I R)$ and accumulated infection risk $(A I R)$ at time $t$ were calculated as follows:

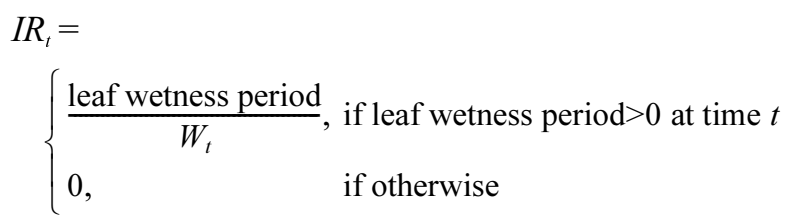

$$
\begin{aligned}
& A I R_{t}= \\
& \begin{cases}A I R_{t-1}+I R_{t}, & \text { if rainfall }<4 \mathrm{~mm} \text { and accumulated } \\
& \text { dry hour }<6 \mathrm{hr} \text { at time } t \\
0, & \text { if otherwise }\end{cases}
\end{aligned}
$$

where $W_{t}$ is the wetness period required for $10 \%$ appressorium formation at time $t$ calculated from the infection model $W(P, T)$. The $A I R_{t}$ was assumed to be zero if rainfall was more than $4 \mathrm{~mm} / \mathrm{hr}$ or a dry period continued for more than or equal to 6 hours. The limiting condition of rainfall was adopted from an observation on rice blast fungus, which showed spore wash-off from leaves and decrease in spore dispersal when rainfall of more than $4 \mathrm{~mm} / \mathrm{hr}$ occurred in the field (Suzuki, 1975). As for the continuous dry period, the 6-hour limit was arbitrarily used assuming that spores may not survive after 6 hours of continuous dry period after germination.

\section{Results}

Appressorium formation. Temporal progressions of the percent appressorium formation of $C$. acutatum under different temperature and wetness period conditions were shown in Fig. 1. Germinated conidia started to form appressoria after 12 hours of wetness at $20^{\circ} \mathrm{C}$, whereas appressoria were observed after 6 hours of wetness at other temperature conditions. Especially, at $30^{\circ} \mathrm{C}$ and $33^{\circ} \mathrm{C}, 18 \%$ and $9 \%$ of conidia, respectively, formed appressoria after 6 hours of wetness without lag period observed. The percent appressorium formation reached approximately 30-40\% at $23-30{ }^{\circ} \mathrm{C}$ after $15-20$ hours of wetness, whereas it was less than $20 \%$ at $20^{\circ} \mathrm{C}$ and $33^{\circ} \mathrm{C}$. In general, the results suggested that temperature affected the length of lag period prior to initiation of appressorium formation and the maximum percent appressorium formation. The lengths of lag period were almost the same at $23^{\circ} \mathrm{C}, 25^{\circ} \mathrm{C}$ and $28^{\circ} \mathrm{C}$, but the maximum percent appressorium formation increased as temperature increased. At all temperature conditions, the percent appressorium formation reached close to its asymptotic values after 18 or 24 hours of wetness period.

Linear infection model. The linear regression analysis resulted in the first order model as follows:

$$
P=-13.3+0.612 T+0.928 W
$$

where $P, T$, and $W$ are the percent appressorium formation, temperature $\left({ }^{\circ} \mathrm{C}\right)$, and wetness period $(\mathrm{hr})$, respectively. The coefficient of determination $\left(R^{2}\right)$ was 0.60 , indicating that $60 \%$ of variation in the percent appressorium formation was accounted for by temperature and wetness period. From Eq. 
(8), the linear infection model to determine wetness periods required for a certain level of appressorium formation at a given temperature was derived as the following equation:

$$
W=\frac{13.3+P}{0.928}-0.659 T
$$

Nonlinear infection model. The nonlinear regression analysis on the percent appressorium formations at each temperature resulted in the logistic models with different parameter estimates (Fig. 1). In all cases, the convergence criterion of nonlinear regression was met, indicating that the parameter estimates were unique solutions. The logistic models for all temperatures appeared to fit the appressorium data well enough to adequately describe the progressions of percent appressorium formation over wetness periods.

By fitting the simplified Equilibrium Model (Eq. 3) to the asymptote values at different temperatures in Fig. 1, the temperature function for $A$ was obtained (Fig. 2). From the series of nonlinear regression analyses by varying $T_{e q}$ in Eq. 3 from $293.15 \mathrm{~K}\left(20.0^{\circ} \mathrm{C}\right)$ to $313.15 \mathrm{~K}\left(40.0^{\circ} \mathrm{C}\right)$ at $1.0 \mathrm{~K}$ intervals, the best temperature function for $A$ was found when $T_{e q}$ was set to $305.15 \mathrm{~K}\left(32.0^{\circ} \mathrm{C}\right)$ based on the $\mathrm{F}$ value and coefficient of determination $\left(R^{2}\right)$. According to the temperature function in Fig. 2, the percent appressorium formation increased as temperature increased up to $28.9^{\circ} \mathrm{C}$ and then decreased rapidly.

Nonlinear fitting of the reciprocal equations, Eqs. (4) and (5) to the estimates of $B$ and $k$ at different temperatures resulted in the temperature functions for $B$ and $k$ in Fig. 3. The responses of $B$ and $k$ to temperature changes were similar to each other, showing fast decrease until $23.0^{\circ} \mathrm{C}$ and then gradual decrease to minimal levels at higher temperatures.

By replacing $A, B$, and $k$ in Eq. (1) with the respective temperature functions, the percent appressorium formation $P$ can be described by the following model:

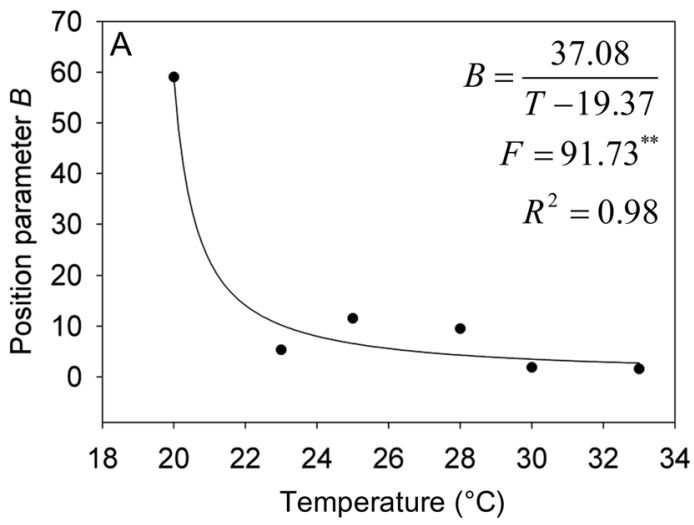

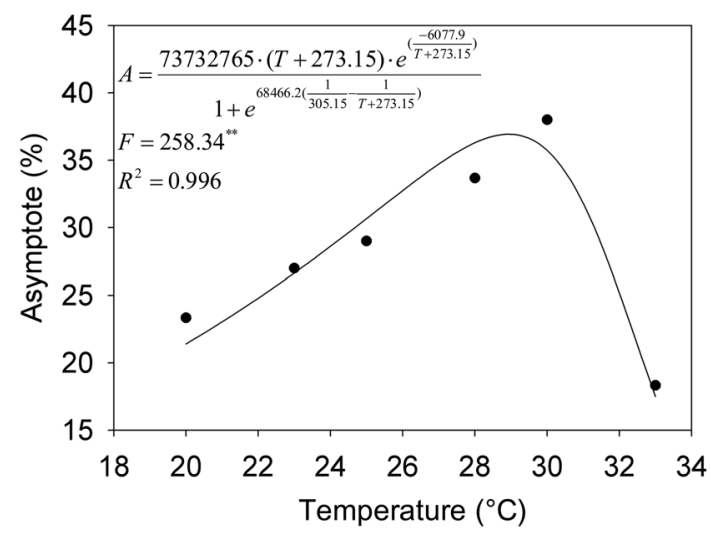

Fig. 2. The maximum percent appressorium formation of $C$. acutatum at different temperatures. The Equilibrium Model for enzymatic reactions was fitted to obtain the temperature function for the parameter $A$ of logistic model.

$$
P(W)=\frac{73732765(T+273.15) e^{\left(\frac{-6077.9}{T+273.15}\right)}}{\left(1+e^{68466.2\left(\frac{1}{305.15}-\frac{1}{T+273.15}\right)}\right)\left(1+e^{\left(\frac{37.08}{T-19.37}-\frac{3.26}{T-19.13} W\right)}\right)}
$$

From Eq. (10), the nonlinear infection model to determine wetness periods required for appressorium formation at certain temperatures was derived as the following equation:

$$
\begin{aligned}
& W(T)=\{B-\ln (A / P-1)\} / k \\
& =\left\{\frac{37.08}{T-19.37}-\ln \left(\frac{73732765(T+273.15) \cdot e^{\left(\frac{-6077.9}{T+273.15}\right)}}{1+e^{68466.2\left(\frac{1}{305.15}-\frac{1}{T+273.15}\right)}} \cdot \frac{1}{\mathrm{P}}-1\right)\right\} \cdot \frac{T-19.13}{3.26}
\end{aligned}
$$

where $P$ is a level of percent appressorium formation, and $T$ is the mean temperature $\left({ }^{\circ} \mathrm{C}\right)$ during wetness period. The response surface of Eq. (11) is presented as the contour projection of the percent appressorium formation with respect to the temperature and wetness period conditions in

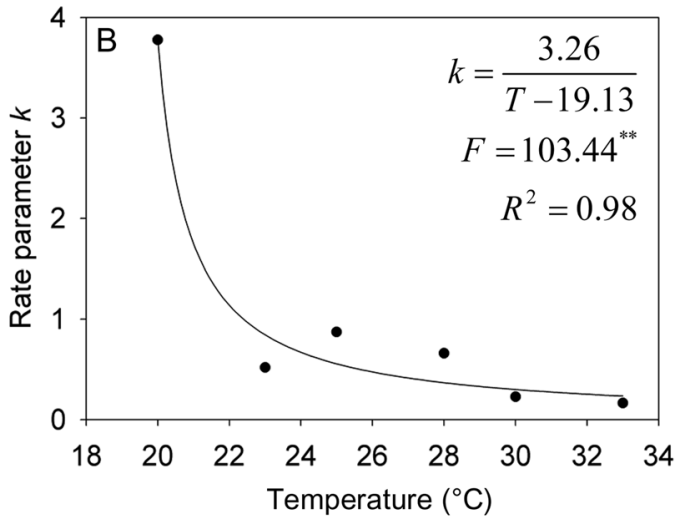

Fig. 3. The estimates of position $(B)$ and rate $(k)$ parameters of the logistic model for the percent appressorium formation at each temperature $(T)$. The reciprocal model was fitted to obtain the temperature functions for the parameters $B$ and $k$ of logistic model. 


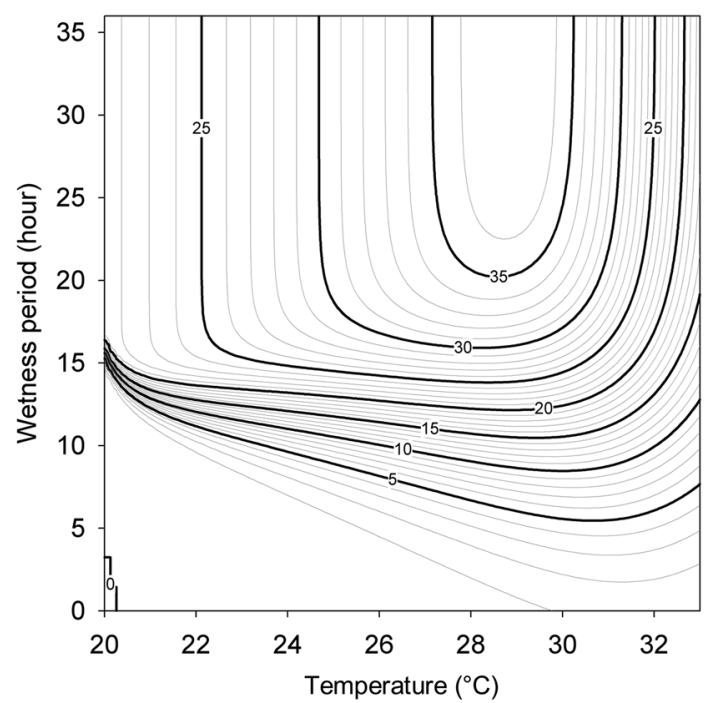

Fig. 4. The contour projection of the combined effects of temperature and wetness period on the percent appressorium formation of C. acutatum.

Fig. 4. The contour projection shows that the optimum temperature for appressorium formation ranged between $28^{\circ} \mathrm{C}$ and $30^{\circ} \mathrm{C}$. It also shows that the percent appressorium formation was more responsive to temperature than wetness period.

Model evaluation. Aptness of the linear and nonlinear infection models in describing the temporal progression of percent appressorium formation was compared in terms of RMSE of the differences between the observed and estimated percent appressorium formation (Table 1). At all temperature conditions tested in this study, the nonlinear infection model resulted in lower RMSE than the linear infection model, indicating that the nonlinear infection model was more appropriate than the linear infection model for describing the temporal progressions of percent appressorium formation. Especially, RMSE of the linear model was substantially high at $20^{\circ} \mathrm{C}, 30^{\circ} \mathrm{C}$, and $33^{\circ} \mathrm{C}$.

Table 1. Comparison between the root mean square errors (RMSE) of the linear model (Eq. 8) and the nonlinear model (Eq. 10) for the effects of wetness period on the percent appressorium formation of C. acutatum at different temperatures

\begin{tabular}{ccc}
\hline \hline \multirow{2}{*}{ Temperature $\left({ }^{\circ} \mathrm{C}\right)$} & \multicolumn{2}{c}{ Root mean square error (RMSE) } \\
\cline { 2 - 3 } & Linear model & Nonlinear model \\
\hline 20 & 74.308 & 8.849 \\
23 & 18.794 & 14.500 \\
25 & 34.449 & 12.261 \\
28 & 50.947 & 39.024 \\
30 & 73.951 & 28.134 \\
33 & 152.148 & 8.569 \\
\hline
\end{tabular}
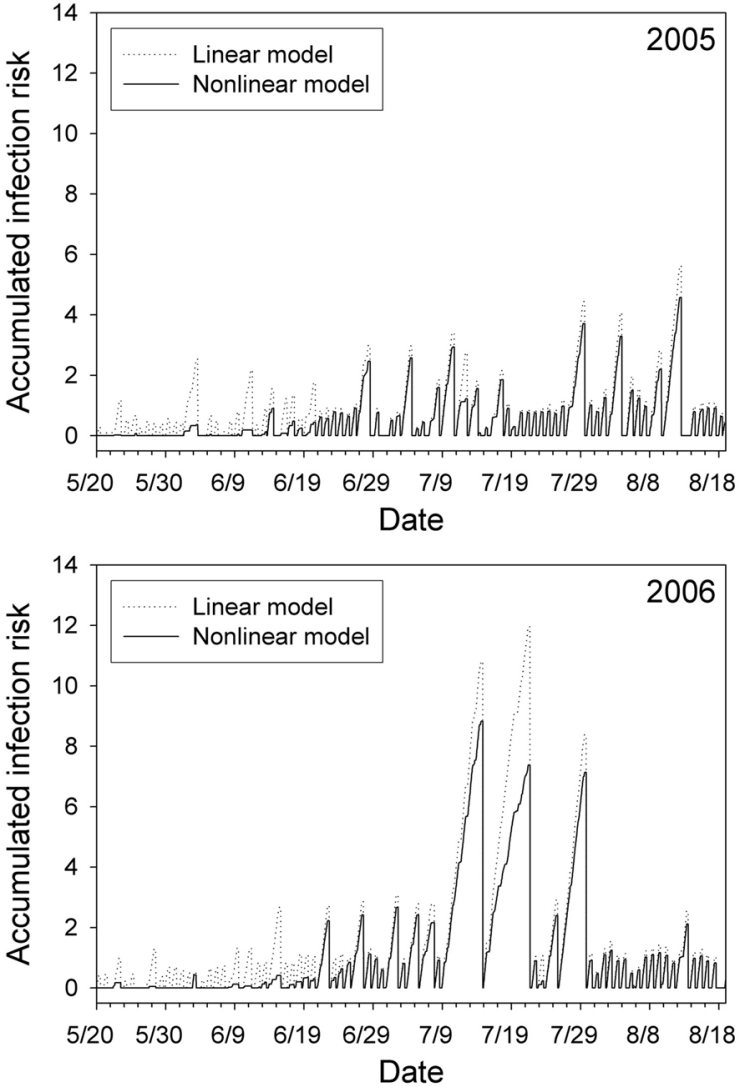

Fig. 5. The accumulated infection risks of anthracnose calculated by the linear infection model (Eqs. 6, 7, and 9) and the nonlinear infection model (Eqs. 6, 7, and 11) using the hourly temperature and wetness period monitored by an automated weather station installed at the experimental pepper field in Asan, Korea in 2005 and 2006.

The possible levels of infection risks forecast by the linear and nonlinear infection models were compared using the hourly temperature and wetness period data monitored by an automated weather station which was installed at an experimental pepper field in 2005 and 2006 (Fig. 5). Although the temporal trends of accumulated infection risks forecast by the two models were similar to each other, the linear infection model always resulted in higher infection risks than the nonlinear infection model throughout the growing seasons. The differences in accumulated infection risks between the linear and nonlinear infection models were apparently large when temperature was relatively low in the early seasons in both 2005 and 2006, and during July in 2006 when frequent rainfall occurred under favorable temperature conditions.

\section{Discussion}

A logistic model for describing combined effects of both temperature and wetness period on appressorium formation 
was developed using laboratory data on percent appressorium formation of $C$. acutatum. In addition, the possible use of the logistic model for forecasting infection risks was also evaluated as compared with a first-order linear model. In this study, we were able to obtain the logistic model with both temperature and wetness period as independent variables by replacing the parameters $A, B$, and $k$ of logistic equation with the respective temperature functions. By having both temperature and wetness period as independent variables, the nonlinear logistic model can be used to determine the length of wetness periods required for certain levels of appressorium formation under different temperature conditions. On the contrary, most of nonlinear models describing the response of plant pathogens in the literature reflect effect of either temperature or wetness period but not both (Carisse et al., 1993; Duthie, 1997; Magboul et al., 1992). Polynomial equations are commonly fitted to empirical data by ordinary least squares methods to determine response surface for describing combined effects of both wetness period and temperature on disease components (Bulger et al., 1987; Schuh, 1991). However, it is often difficult to explain biological significance of parameter estimates in polynomial models (Scherm and van Bruggen, 1993).

The results in Figs. 1 and 2 suggested that temperature affected the length of lag period prior to appressorium formation and the maximum percent appressorium formation. The lag period of longer than 10 hours was necessary to initiate appressorium formation at low temperature $\left(20^{\circ} \mathrm{C}\right)$, and the upper limit of percent appressorium formation was decreased when temperature became the suboptimum range. Based on the length of lag period and the maximum percent appressorium formation, $28-30^{\circ} \mathrm{C}$ appeared to be the optimum temperature for appressorium formation of $C$. acutatum in this study.

The simplified Equilibrium Model of Eq. (3) appeared suitable for describing the effects of temperature on the estimates of maximum appressorium formation $(A)$. The response of upper limit of appressorium formation to temperature in Fig. 2 shows the typical temperature response of enzymatic reactions in the cells (Peterson et al., 2004). In general, as the temperature is increased within a given range, the rate of enzymatic reactions increases up to the optimal temperature. Above this point, protein denaturation sets in and cell functions such as appressorium formation fall sharply to zero. With regard to the parameter estimates of $B$ and $k$ in Fig. 3, the reciprocal models were appropriate to fit the empirical data on temperature-dependent estimates of the parameters. The high estimates of $B$ and $k$ at $20^{\circ} \mathrm{C}$ were caused by the abrupt increase in appressorium formation at $20^{\circ} \mathrm{C}$ after 18 hours of wetness.

The response surface of appressorium formation to the combined effects of temperature and wetness period is shown as the contour plot in Fig. 4. The contour plot can be used to determine the minimum requirement of temperature and wetness period for a certain level of appressorium formation. Since appressorium formation is a prerequisite for penetration into host plants by $C$. acutatum, the levels of percent appressorium formation could be considered the infection risk levels. The contour plots defining the combined effects of temperature and wetness period on plant infection by fungal pathogens were commonly used as the infection models for disease forecasting (Bulger et al., 1987; Schuh, 1991).

The RMSE is a measure of how good fit a model makes to the observed data (Kim et al., 2005; Workneh and Rush, 2006). Based on RMSE, the nonlinear model of Eq. (10) fitted the observed data on temporal progression of appressorium formation better than the linear model of Eq. (8), and the linear model resulted in significantly under- and over-estimation of response at low and high temperatures, respectively. Compared with the nonlinear infection model of Eq. (11), the linear infection model always predicted a shorter wetness period for appressorium formation. Consequently, as shown in Fig. 5, the accumulated infection risks forecast by the linear infection model were always higher than those by the nonlinear infection model. The significantly high accumulated infection risks forecast by the linear model in the early seasons of both 2005 and 2006 were caused by the nature of linear model under-estimating wetness periods for appressorium formation at low temperatures. The high levels of accumulated infection risk during July in 2009 resulted from continuation of favorable weather conditions for fungal infection due to frequent rainfall.

In this study, a logistic model was proposed to evaluate the response of appressorium formation of C. acutatum to the combined effects of temperature and wetness period. By replacing the parameters of logistic model with respective temperature functions, both temperature and wetness period were included as independent variables in the logistic model. Consequently, we were able to develop a nonlinear infection model determining the infection risks based on hourly temperature and wetness period. Most of infection models in the literature are polynomial models whose parameters may not contain clear biological significance (Scherm and van Bruggen, 1993). The temperature functions for the parameters of logistic model in this study represent the biological responses of $C$. acutatum to temperature, shaping the temporal progression curve of appressorium formation. In practice, the nonlinear infection model could be used to determine fungicide spray schedule to control anthracnose of pepper in the field. Effectiveness of the nonlinear infection model in reducing fungicide sprays needs to be evaluated in the future. 


\section{Acknowledgements}

This work was supported by a grant of Special Agriculture Program (800220040013000) from Rural Development Administration, Republic of Korea. We thank H. T. Kim at Chungbuk National University, Republic of Korea for providing the isolate of Colletotrichum acutatum used in this work.

\section{References}

Anonymous. 2008. Application Guide of Agricultural Chemicals. Korea Crop Protection Association. Seoul, Korea. 1080 pp.

Biggs, A. R. and Northover, J. 1988. Influence of temperature and wetness duration on infection of peach and sweet cherry fruits by Monilinia fructicola. Phytopathology 78:1352-1356.

Bulger, M. A., Ellis, M. A. and Madden, L. V. 1987. Influence of temperature and wetness duration on infection of strawberry flowers by Botrytis cinerea and disease incidence of fruit originating from infected flowers. Phytopathology 77:1225-1230.

Campbell, C. L. and Madden, L. V. 1990. Introduction to plant disease epidemiology. John Wiley \& Sons, Inc. 531 pp.

Carisse, O., Kushalappa, A. C. and Cloutier, D. C. 1993. Influence of temperature, leaf wetness, and high relative humidity duration on sporulation of Cercospora carotae on carrot leaves. Phytopathology 83:338-343.

Cho, E. K., Park, K. S., Yang, S. S. and Lee, E. J. 1987. Studies on development of forecasting method for red-pepper anthracnose. In: Annual Research Report (Biology Division), ed. by Agricultural Science Institute, pp. 351-355. Agricultural Science Institute. Suwon, Korea.

Duthie, J. A. 1997. Models of the response of foliar parasites to the combined effects of temperature and duration of wetness. Phytopathology 87:1088-1095.

Evans, K. J., Nyquist, W. E. and Latin, R. X. 1992. A model based on temperature and leaf wetness duration for establishment of Alternaria leaf blight of muskmelon. Phytopathology 82:890895.

Freeman, S., Katan, T. and Shabi, E. 1998. Characterization of Colletotrichum species responsible for anthracnose diseases of various fruits. Plant Dis. 82:596-605.

Hau, B., Eisensmith, S. P. and Kranz, J. 1985. Construction of temporal models. In: Mathematical Modeling of Crop Disease, ed. by C. A. Gilligan, pp. 31-65. Academic Press. New York, USA.

Hong, J. K. and Hwang, B. K. 1998. Influence of inoculum density, wetness duration, plant age, inoculation method, and cultivar resistance on infection of pepper plants by Colletotrichum coccodes. Plant Dis. 82:1079-1083.

Kang, B. K., Min, J. Y., Kim, Y. S., Park, S. W., Bach, N. V. and Kim, H. T. 2005. Semi-selective medium for monitoring Col- letotrichum acutatum causing pepper anthracnose in the field. Res. Plant Dis. 11:21-27.

Kim, C. H. 1993. Current status of fungal and bacterial diseases of hot pepper and their control measures. J. Korean Capsicum Research Cooperative 2:1-11.

Kim, C. H. and Park, K. S. 1988. A predictive model of disease progression of red-pepper anthracnose. Korean J. Plant Pathol. 4:325-331.

Kim, K. S., Wang, T. C. and Yang, X. B. 2005. Simulation of apparent infection rate to predict severity of soybean rust using a fuzzy logic system. Phytopathology 95:1122-1131.

Lalancette, N., Ellis, M. A. and Madden, L. V. 1988. Development of an infection efficiency model for Plasmopara viticola on American grape based on temperature and duration of leaf wetness. Phytopathology 78:794-800.

Madden, L. V., Yang, X. and Wilson, L. L. 1996. Effects of rain intensity on splash dispersal of Colletotrichum acutatum. Phytopathology 86:864-874.

Magboul, A. M., Geng, S., Gilchrist, D. G. and Jackson, L. F. 1992. Environmental influence on the infection of wheat by Mycosphaerella graminicola. Phytopathology 82:1407-1413.

Manandhar, J. B., Wang, T. C. and Hartman, G. L. 1995. Anthracnose development on pepper fruits inoculated with Colletotrichum gloeosporioides. Plant Dis. 79:380-383.

Park, K. S. and Kim, C. H. 1992. Identification, distribution, and etiological characteristics of anthracnose fungi of red pepper in Korea. Korean J. Plant Pathol. 8:61-69.

Peres, N. A., Timmer, L. W., Adaskaveg, J. E. and Correll, J. C. 2005. Lifestyles of Colletotrichum acutatum. Plant Dis. 89:784-796.

Peterson, M. E., Eisenthal, R., Danson, M. J., Spence, A. and Daniel, R. M. 2004. A new intrinsic thermal parameter for enzymes reveals true temperature optima. J. Biol. Chem. 279:20717-20722.

SAS Institute Inc. 1985. SAS User's Guide: Statistics. SAS Institute Inc., Cary, NC. 956 pp.

Scherm, H. and van Bruggen, A. H. C. 1993. Response surface models for germination and infection of Bremia lactucae, the fungus causing downy mildew of lettuce. Ecol. Model. 65:281-296.

Schuh, W. 1991. Influence of temperature and leaf wetness period on conidial germination in vitro and infection of Cercospora kikuchii on soybean. Phytopathology 81:1315-1318.

Shin, H. J., Chen, Z. J., Hwang, J. and Lee, S. G. 1999. Comparison of pepper anthracnose pathogen from Korea and China. Plant Pathol. J. 15:323-329.

Suzuki, H. 1975. Meteorological factors in the epidemiology of rice blast. Annu. Rev. Phytopathol. 13:239-256.

Workneh, F. and Rush, C. M. 2006. Weather factors associated with development of sorghum ergot in the Texas Panhandle. Plant Dis. 90:717-722. 